\title{
Creative Product Design Based on Dong Culture Creative
}

\author{
Yang Shuo ${ }^{1}$ and ZhangChao ${ }^{2 *}$ \\ ${ }^{1}$ Guizhou University, Guiyang, Guizhou, 550025, China \\ ${ }^{2}$ Guizhou University, Guiyang, Guizhou, 550025, China
}

\begin{abstract}
The study of Dong ethnic cultural resources provides the experience basis and method path for the designing and development of cultural and creative products with Dong ethnic-cultural characteristics. In the research, first of all, the Dong culture is mainly taken as the core and the Dong cultural resources are classified. Secondly, it studies the attributes of cultural and creative products and designs and combs the cultural and creative products of Dong nationality. Furthermore, the creative product design model of Dong ethnic culture is constructed, and eye tracking technology is taken as an example to accurately extract the cultural identification characteristics of cultural materials through modern science and technology, and the specific case design is carried out. Through the excavation, arrangement, and induction of Dong ethnic cultural resources, the attributes of cultural and creative products are analyzed, and the design model is constructed to provide ideas for the design of Dong ethnic cultural and creative products, which is conducive to enhancing the value of Dong ethnic culture and cultural and creative products and provides references for the cultural development of other ethnic minorities.
\end{abstract}

\section{Introduction}

With such China's rapid economic growth, cultural and creative industries, as an emerging industry, have demonstrated great development potential in promoting the integration of regional economic and improving the development of ethnic minority areas[1]. Through the research on the cultural and creative industries of ethnic minorities, we will continuously explore new paths, create new models, and derive new forms, which will play a significant role in promoting the rapid formation of a new ethnic economic system [1]. National culture shapes individual psychology and group locality, nationality, and national characters. Enhancing the modern design level of national culture is the responsibility of researchers, and protecting as well as inheriting traditional culture is a matter of urgent priority[2]. This study aimed to excavate, explore, and promote the Dong ethnic culture[3]. Furthermore, with an in-depth understanding of Dong, it is possible to better design and develop cultural and creative products, thereby greatly improving the national brand quality and unique charm. Making perfect national cultural creative products provides a powerful impetus for promoting the development of national cultural industry and tourism.

Utilizing the innovative research perspective to provide references for the development of cultural soft power in minority areas[4], which also improve the comprehensive strength of ethnic minority regional culture.

\section{Design Thinking of Dong Ethnic Culture}

\subsection{The Current Situation of Dong Ethnic Culture and Cultural Creative Products}

Throughout history, the wise Dong people have created a rich national culture. Among them, architecture, clothing, cuisine, etiquette, festival activities, folk arts, and crafts are all extremely ethnic[5]. However, ethnic songs and dances as well as national arts are derived from nature and have many mysterious characteristics, which provide huge design raw materials for the design of cultural and creative products. From the perspective of history and culture, the cultural characteristics of Dong nationality reflect the traditional Chinese aesthetic art and integrate cultural connotation. Meanwhile, under the economic advancement, cultural industries have increasingly received the attention of the government, attracting a large number of tourists to consume in ethnic minority areas. Therefore, a rich culture has created a positive economic market. At present, there are still some problems in the field of design and development of Dong cultural and creative products, including insufficient cultural connotation, empty regional characteristics, single product types, inadequate quality, weak innovative design, etc. In summary, improving existing problems is of great significance to the progress of creative product design methods for regional culture. 


\subsection{Design Thinking of Dong Ethnic Cultural and Creative Products}

Based on the current situation of Dong ethnic regional culture and cultural creative product market. The previous research and analysis efforts of Dong nationality are indicated that the products lack national souls. Owing to this, it is necessary to characterize the ethnic-cultural resources of Dong and establish a resource classification model to assist creative design, and the representative ethnic cultural elements simultaneously integrated with modern technology to enhance the visual characteristics in this study. Finally, this study validates the specific design case to further improve the quality and value of Dong's cultural and creative products (Fig. 1).

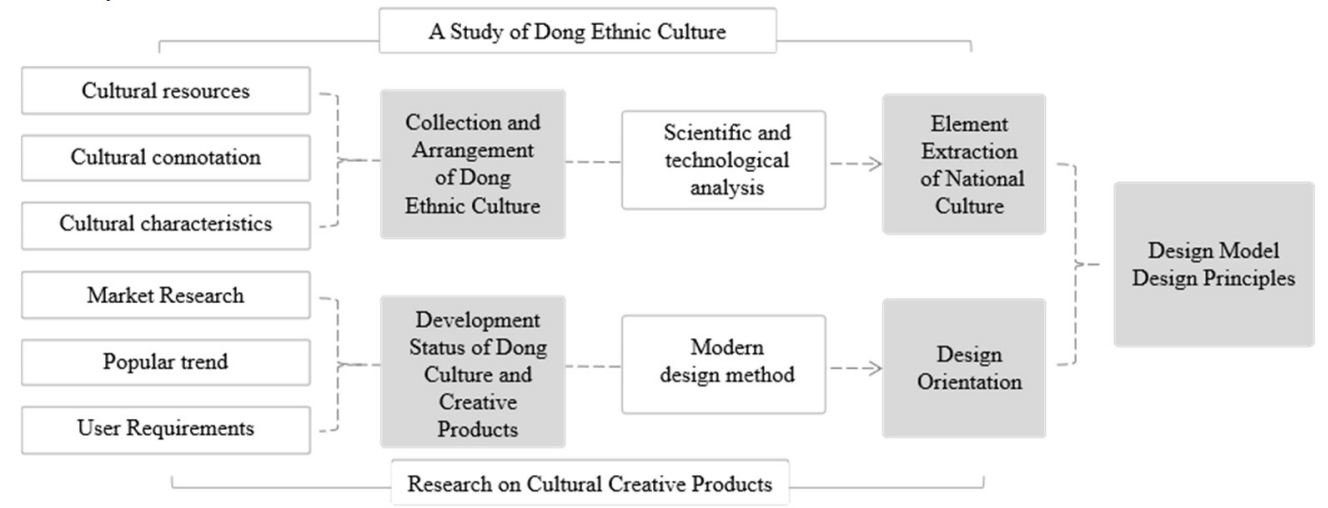

Fig 1. Research thinking

\section{Creative product design model based on Dong culture}

\subsection{Dong Ethnic Cultural Resources and Modal Classification}

Dong nationality's diverse ethnic culture is involving various cultural contents and forms. Therefore, it is necessary to collect, sort out and classify Dong's cultural resources, characteristics, connotation, prototypes, symbols, etc., thus to realize the demands of quickly obtaining regional cultural features and characteristics and then assist the development of the creative design. This paper divides Dong ethnic culture into three levels: material culture, behavioral culture, and spiritual culture. Therefore, correspond and summarize different types of Dong cultural forms and contents (Fig. 2). Meanwhile, matching the psychological cognitive level put forward by Donald A- Norman and conforming to the laws of design thinking, which is friendly for designers to translate regional cultural information[6]. Seeking cultural elements, cultural prototypes and cultural symbols with distinctive national characteristics to sublimate design creativity, so as to highlight the uniqueness and national characteristics of products. Based on that, focusing on the symbolic meaning of culture, designs and refines the cultural prototype with far-reaching regional cultural connotation, embodies the symbolic meaning and value of culture in creative design, as well as better highlights the regional features and charm of products.

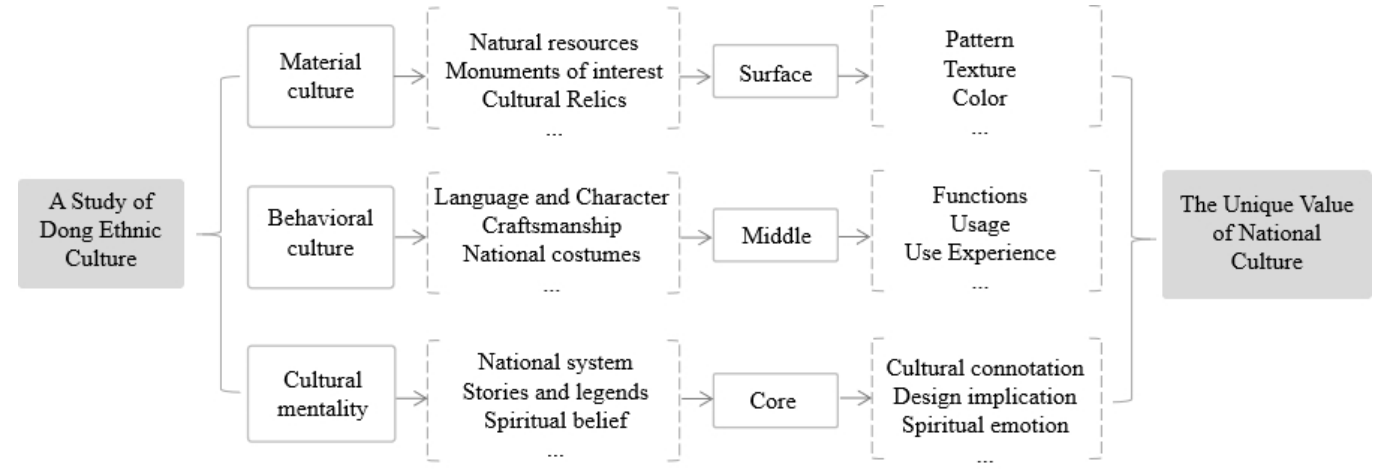

Fig 2. Modal classification of Dong ethnic cultural resources

\subsection{Design Elements and Representation of Dong Cultural Creative Products}

The designing process usually researches cultural materials, i.e. their shapes, forms, colors, materials, textures, emotional experiences, etc. According to Norman's three levels of emotional cognition, the design attributes and elements of the product are summarized hierarchically to form a design element representation framework system corresponding to design and culture (Fig. 3). Cultural and creative products will eventually be characterized mainly by function, structure, technology, form, color, material, decoration, texture, etc. The hierarchical division framework and the structural division of the above cultural levels can correspond to 
each other, which is conducive to better combination with cultural resources in the process of design representation, thus better interpreting cultural themes.

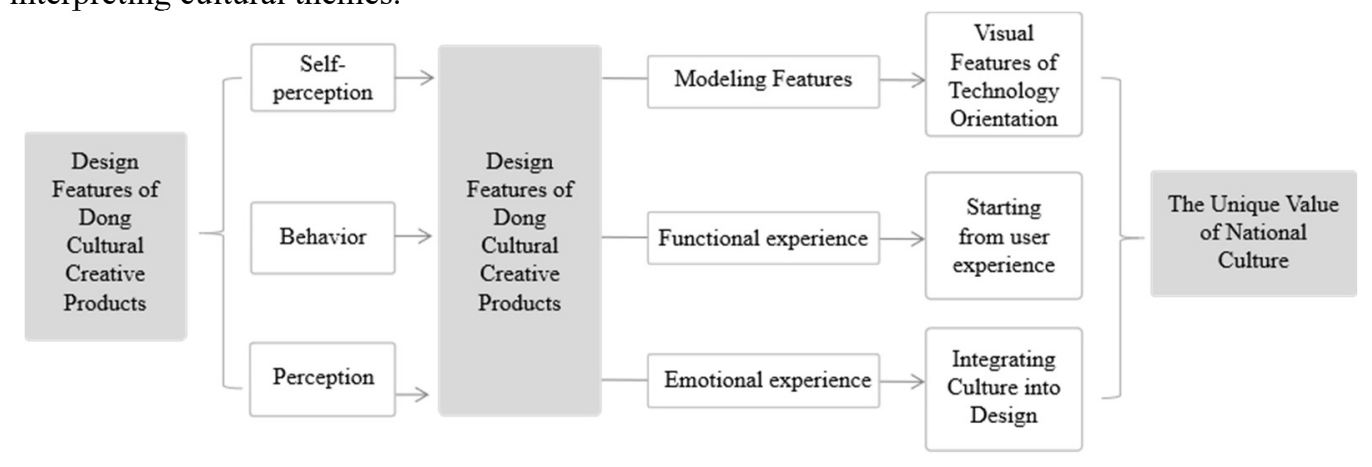

Fig 3. The design attributes of cultural creative products

\section{Design and Application Combined with Eye Tracking Technology}

Based on the investigation of Dong's cultural resources and the analysis of hierarchical modes, as the content element, Dong's Drum Tower is a unique intangible cultural heritage with a series of functions such as religion, folk customs, and laws, also carrying the cultural memory of Dong people. Relying on the visual experiment of eye tracking technology[7], the modeling features of Dong Drum Tower can be positioned, and the design path is verified via the display[8] and application of related cases.

\subsection{Experimental process}

The experiment is based on the building body and various structural parts of Dong's Drum Tower (Fig. 4). First of all, the black-and-white treatment of the photos taken on the spot to avoid the influence of color. Secondly, the processed photo $\mathrm{A}$ is taken as a test sample for identification experiments. To refrain the influence of directed thinking, the local structures of each drum tower are decomposed and put together for testing (B1, B2, B3, $\mathrm{B} 4)$. The fixation time of the two groups is $60000 \mathrm{~ms}$ respectively, and relevant data were recorded to explore the relationship between the feature types of drum towers and the degree of visual recognition.

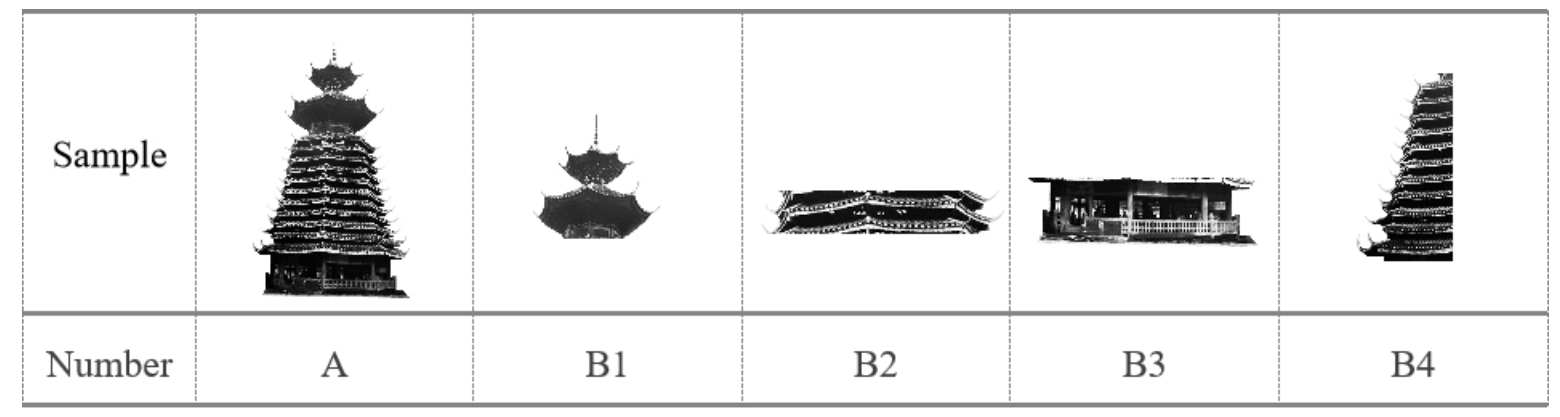

Fig 4. Visual samples of the experimental sample

\subsection{Experimental Instruments and Objects}

When the subjects were selected, 20 school personnel (aged 18-60 years old, half male and half female) who did not study the samples were selected, and with no color weakness or color blindness, as well as their eyesight was guaranteed to be above 1.0. The experiment was carried out in the man-machine laboratory, and the subjects remained comfortable without interference. The "The Eye Tribe" eye tracking instrument was used in the experiment. The sampling rate was $60 \mathrm{~Hz}$, the average accuracy was 0.5 degrees, the spatial resolution was 0.1 degrees (RMS), and the delay time was less than $20 \mathrm{~ms}$.

\subsection{Analysis of experimental data}

Through the dynamic capture of the visual changes of the subjects by the eye tracking instrument, the density hot area and residence time of the subjects' attention, the degree of attention preference of the subjects and the eye movement trajectories are directly reflected in the form of the heat map and track map, while the primary colors of the samples indicate that they have not been paid attention to. According to the subjects' attention heat map and visual track map (Fig. 5), combined with the samples of the whole and local structures, the recognition degree in the drum tower shape is in turn: building body $>$ cornices $>$ tower top $>$ bottom. The mysterious veil of drum tower culture is gradually clearing and providing a precise 
development direction for the series of drum tower cultural industries.
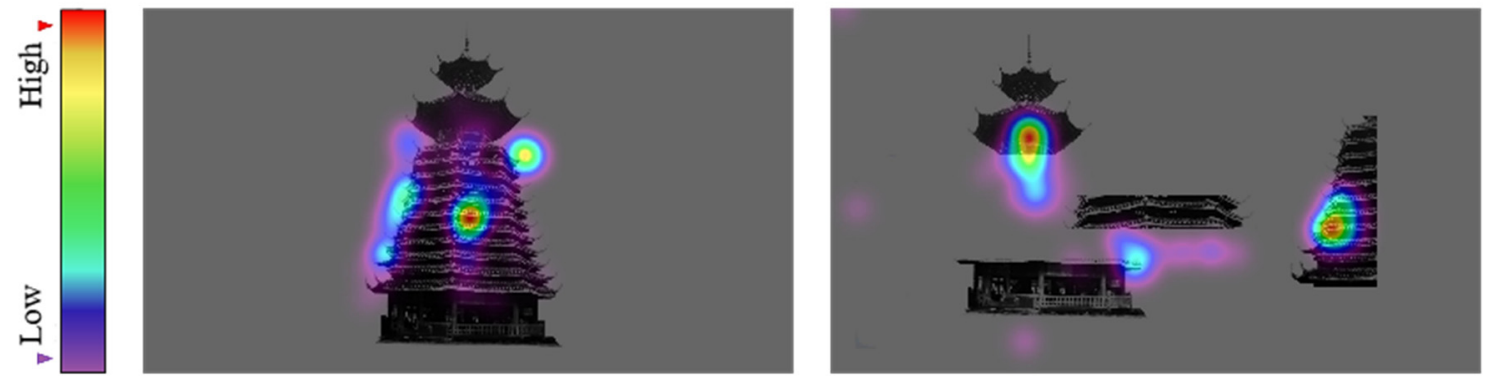

Fig 5. Presentation form of heat maps and track maps, and the heat maps of experimental samples

\subsection{Design practice}

Through the accurate analysis of the identification characteristics of Dong Drum Tower, the three main characteristics of the building body, cornices, and treasure roof are refined and optimized, and the contemporary aesthetic concept is integrated based on paying attention to user experience. By feature extraction, the combination of portable humidifier and dehumidifier is made(Fig. 6). In the new design, the overall shape is designed according to the characteristics of the experimental structure, by using two classical colors of traditional Chinese white and black to rich Dong ethnic cultural characteristics into the level of modern aesthetic feeling. In the functional part, on the premise of safety and energy conservation, the cornices are inlaid with luminous light belts, which are environmentally friendly and humanized. Fog, set off by the lamp belt, creates an elegant dynamic aesthetic feeling, which improves the viewing feeling during the using process of consumers (Fig. 7). The mysterious Drum Tower is surrounded by fog and seems to be in nature. By reasonable design models, brand-new cultural and creative products are designed, as well as people's perception and memory of the cultural characteristics of the Dong Drum Tower are added, thus spreading Dong culture to all corners of the world.

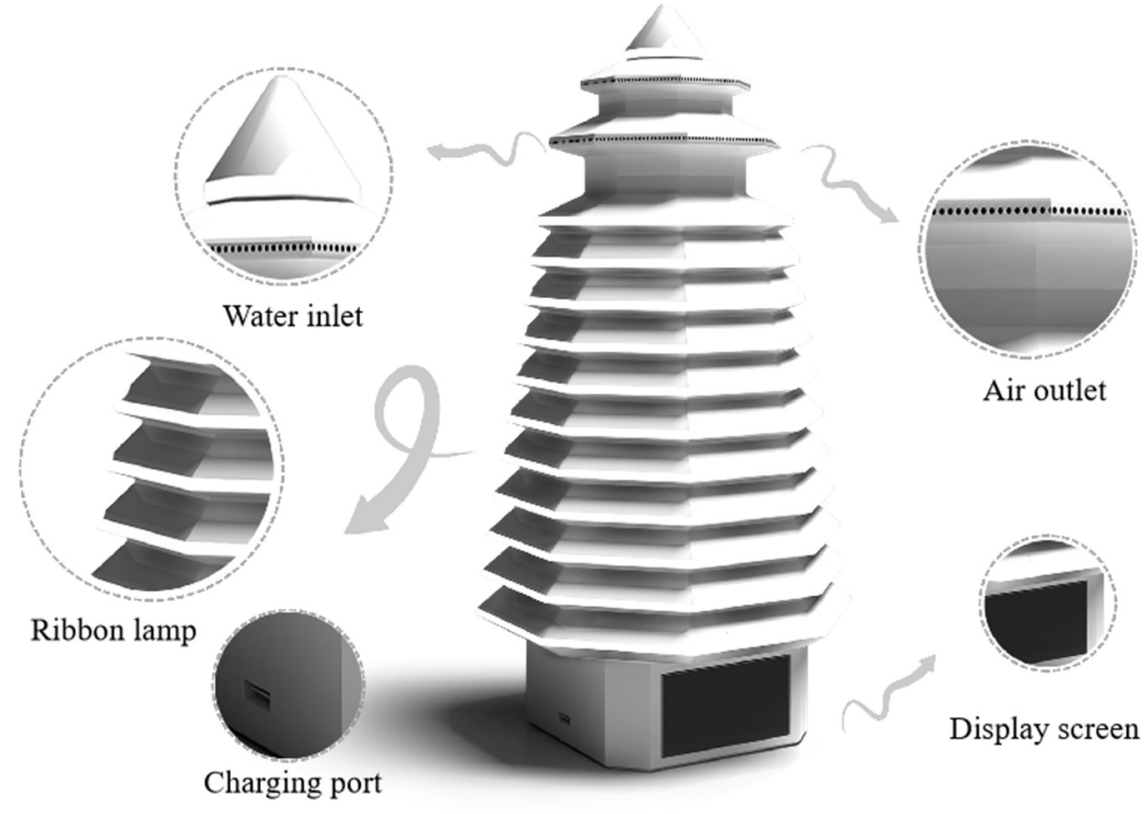

Fig 6. Cultural creative product design
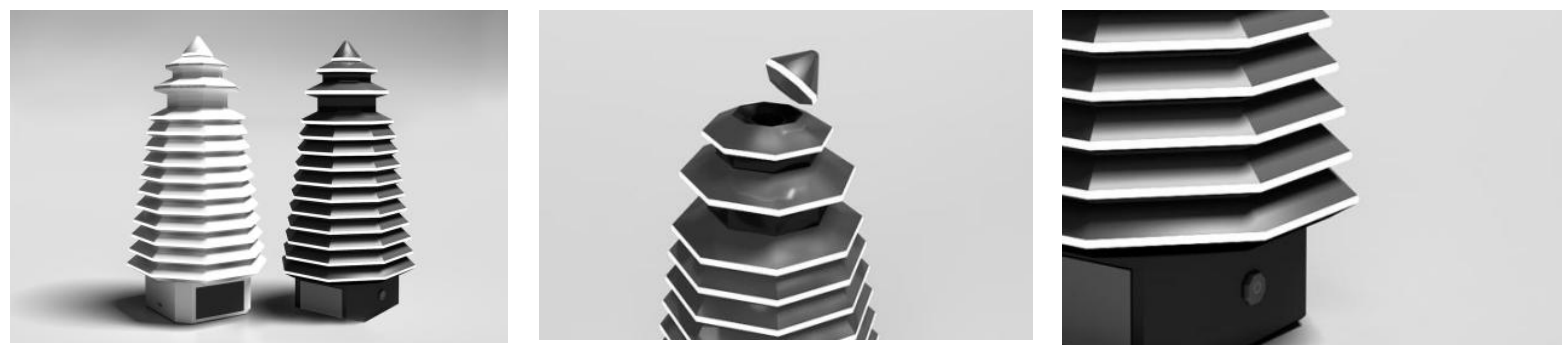

Fig 7. Detailed design drawings 


\section{Summary}

Taking Dong nationality as an example, this paper puts forward the design method path through systematic combing of Dong nationality culture and cultural and creative products. Combined with eye tracking technology, the accuracy of models in brand positioning is improved. On the basis of this, a designed model is established which hoping to help designers involve more cultural and creative products with Dong ethnic cultural characteristics. It provides a basis for the development of Dong cultural products and a reference for the development of cultural industries in other regions. This study can provide a new approach for the development of ethnic cultural industries, improve the local economic level, enhance the identity of ethnic minority compatriots to regional culture and the cultural confidence of the whole people, and ultimately contribute to realizing the vigorous development of the cultural industry.

\section{Acknowledgments}

Funding: This project was supported by the National Social Science Foundation of China(grant numbers: 11CG126). The Humanities and Social Science Foundation of Guizhou Provincial Department of Education( grant numbers: 2016ssd17).

\section{References}

1. Li, W.Y., \& Z, H.P. (2012). A Study of Stagelization of Ethnic Minority Cultural Symbols from The Perspective of Authenticity. Human Geography, 27(3), 34-38.

2. Zhou, H.P. (2010). Practice and Exploration of China's Intangible Cultural Heritage Protection. Qiushi, 4, 44-46.

3. Li, J. (2010). Villages of Dong Nationality in Zengchong, Cong Jiang County, Southeast Guizhou. Art Panorama, 10: 56-57.

4. Han, Z.Y.(2016). TRIZ Applied in Products Perceptual Form Shaping Process. Industrial Design, 8, 123-124.

5. Zhu, H.Z., \& Zhang, Z.Z.(2005), The Appreciation of the Beautiful of the Dong cultural connotation. Beijing: The Ethnic Publishing House, 75-78.

6. Yang, S., Tang, C.Q., \& Zhang, C. (2020). Research on the Design of Modern Public Facilities with the Characteristics of Dong Drum Tower in Southeast Guizhou. Art Research, 1, 96-97.

7. Scott N, Zhang R, \& Le D, et al. (2019). A review of eye-tracking research in tourism. Current Issues in Tourism, 22(10), 1244-1261.

8. Wen, C.G., Gou, B.C., Wu, L.J., \& Ma, Wei.L.(2018). Extraction and Application Research of Culture Design Gene Based on Eye Movement Analysis. Computer Engineering and Applications, 54(11), 217-224, 235. 